\title{
Ocorrência de neurite em pacientes com hanseníase: análise de sobrevida e fatores preditivos
}

\author{
Occurrence of neuritis among leprosy patients: \\ survival analysis and predictive factors
}

\author{
Soraya Diniz Gonçalves ${ }^{1}$, Rosana Ferreira Sampaio ${ }^{2}$ \\ e Carlos Maurício de Figueiredo Antunes ${ }^{3}$
}

\begin{abstract}
RESUMO
A neurite na hanseníase é responsável pelas deformidades e incapacidades. 0 objetivo desta coorte histórica foi investigar os fatores de risco associados ao tempo até a ocorrência da neurite. Foram acompanhados 595 pacientes, no período de 1993 a 2003 . Empregou-se a técnica de tabela de vida e 0 método de Kaplan-Meier para a curva de sobrevida. Para testar diferenças entre os grupos quanto ao tempo até a ocorrência de neurite, foi usado o log-rank e para estimar as razões de risco, o modelo de regressão de Cox. Pouco mais da metade (54\%) da amostra teve neurite, sendo o principal intervalo de tempo de zero a 11,9 meses. 0 grau de incapacidade na admissão e o índice baciloscópico associaram-se fortemente à ocorrência de neurite, confirmando a necessidade do diagnóstico precoce da hanseníase, bem como do acompanhamento neurológico regular e intervenções adequadas.
\end{abstract}

Palavras-chaves: Hanseníase. Neurite. Fatores de risco.

\begin{abstract}
Neuritis in leprosy cases is responsible for deformities and disability. The objective of this historical cohort study was to investigate the risk factors associated with the time taken for neuritis to occur. This study followed up 595 patients from 1993 to 2003. The life table technique and the Kaplan-Meier method for survival curves were used. The log-rank test was used to test differences between groups regarding the time take for neuritis to occur, and the Cox regression model was used to estimate the hazard ratios. Just over half (54\%) of the sample had neuritis, which had mostly taken 0 to 11.9 months to appear. The degree of disability at admission and the bacillary index were strongly associated with the occurrence of neuritis, thus confirming the need for early diagnosis of leprosy, as well as regular neurological follow-up and appropriate interventions.
\end{abstract}

Key-words: Leprosy. Neuritis. Risk factors.

A hanseníase é identificada, caracterizada e temida pelo seu alto potencial deformante. Seu agente etiológico, o Mycobacterium leprae, é um bacilo hospedeiro intracelular obrigatório (tropismo por células nervosas, bainha de Schwann), podendo provocar inflamações, reações imunológicas e processos compressivos que podem evoluir de uma neuropraxia a uma neurotmese, ou seja, de um dano neural leve e transitório a uma lesão completa e irreversível do nervo. 0 comprometimento dos nervos periféricos é a característica principal da doença podendo, acarretar ao paciente, deficiências e incapacidades, diminuindo sua capacidade de trabalho, limitando sua vida social, tornando-o vítima de estigma e preconceito ${ }^{6} 182021$

A neurite ou neuropatia é reconhecidamente o fator que determina as deformidades que podem levar às incapacidades nesses pacientes. Sua fisiopatologia ainda é pouco conhecida, apesar de várias investigações recentes sobre este tema $a^{1101113151718}$.

As neuropatias refletem o processo inflamatório acompanhado de resposta imune, que pode ocorrer nos nervos periféricos, após a infecção pelo Mycobacterium leprae. 0 monitoramento da função neural, o diagnóstico precoce e as intervenções adequadas são essenciais à preservação da estrutura e função do nervo periférico, contribuindo para evitar as incapacidades ${ }^{3416} 0$ presente estudo teve como objetivo investigar os fatores de risco associados ao tempo até a ocorrência do primeiro episódio de neurite, após iniciar poliquimioterapia, identificando e comparando possíveis preditores de sua ocorrência.

\footnotetext{
1. Curso de Fisioterapia, Pontifícia Universidade Católica de Minas Gerais, Betim, MG. 2. Departamento de Fisioterapia e Terapia Ocupacional, Universidade Federal de Minas Gerais, Belo Horizonte, MG. 3. Departamento de Parasitologia, Universidade Federal de Minas Gerais, Belo Horizonte, MG.

Endereço para correspondência: Prof ${ }^{ \pm}$Soraya Diniz Gonçalves. Rua Souza Magalhães 130/201, Barreiro, 30640-570 Belo Horizonte, MG.

Tel: 5531 9181-5113

e-mail: sorayadinizg@yahoo.com.br

Recebido para publicação em 24/03/2008

Aceito em 01/09/2008
} 


\section{PACIENTES E MÉTODOS}

Esta investigação, uma coorte histórica, estudou uma amostra de 595 pacientes registrados em uma unidade de saúde da Cidade de Belo Horizonte-MG, Brasil, no período compreendido entre 1993 e 2003. Destaca-se que esses pacientes tiveram o diagnóstico de hanseníase definido na referida unidade, receberam tratamento com poliquiomioterapia (PQT) e acompanhamento de fisioterapia, foram avaliados quanto aos aspectos neurológicos no início, durante e no final do tratamento, e tiveram alta por cura (alta medicamentosa).

As variáveis (sociodemográficas e clínicas) incluídas na análise foram: sexo, idade, região de procedência, profissão, forma clínica, ocorrência de neuropatia, reações, acompanhamento fisioterápico, índice baciloscópico na admissão, número de nervos acometidos no início do tratamento, uso e dosagem de prednisona, tempo de tratamento, grau de incapacidade na admissão e na alta. Todas as informações foram obtidas por meio de levantamento dos dados nos prontuários.

A análise do tempo até a ocorrência de neurite e identificação de possíveis preditores para esse evento foi realizada por meio da análise de sobrevivência. Essa é uma técnica empregada quando se deseja estudar o tempo até a ocorrência do evento de interesse, nesse caso a neurite hansênica. Neste estudo, as censuras ocorreram apenas devido ao término do tratamento medicamentoso (censura administrativa), antes do aparecimento da neurite, em alguns pacientes ${ }^{2}$.

Empregou-se a técnica de tabela de vida, para obter a estimativa da função de sobrevida, e o método de Kaplan-Meier, para construção da curva de sobrevida. Para testar diferenças entre os grupos quanto ao tempo, até a ocorrência de neurite, empregou-se o teste de log-rank. Utilizou-se como referência, o mês de tratamento em que ocorreram os episódios de neurite; como tempo zero, considerou-se a data ou mês do diagnóstico.

Para estimar as razões de risco (Hazard ratios) na análise univariada e multivariada, utilizou-se o modelo de regressão de Cox. Considerou-se para entrada das variáveis preditoras no modelo inicial um valor $\mathrm{p}$ de 0,20 e, para permanência da variável no modelo final, o nível de significância de 0,05 . 0 pacote estatístico SPSS (Statistival Package for the Social Sciences) versão 12 foi utilizado para análise dos dados ${ }^{19}$

Este estudo foi aprovado pelo Comitê de Ética da Universidade Federal de Minas Gerais sob o parecer n ${ }^{0}$ ETIC 0163/06.

\section{RESULTADOS}

Dos 595 pacientes, 53,3\% eram do sexo masculino e 46,7\% do feminino. A média de idade foi de 42, $\pm 16,5$ anos. Com relação à ocupação, 21,8\% eram trabalhadores dos serviços e comércio, seguido de trabalhadores de bens e serviços $(19,9 \%)$, donas de casa $(17,9 \%)$, estudantes (11,3\%), aposentados (8,3\%) e o restante incluídos na categoria outros.

Os dados clínicos foram inicialmente estudados de forma descritiva (Tabela 1). Encontraram-se registros referentes às neurites em 529 prontuários. Os dados demonstraram que 53\% da amostra tiveram neurite, 19\% reações hansênicas,

Tabela 1 - Características clínicas da amostra.

\begin{tabular}{|c|c|c|c|c|c|}
\hline \multirow[t]{2}{*}{ Características clínicas } & \multicolumn{2}{|c|}{ Casos } & \multirow[t]{2}{*}{ Características clínicas } & \multicolumn{2}{|c|}{ Casos } \\
\hline & $\mathrm{n}^{0}$ & $\%$ & & $\mathrm{n}^{0}$ & $\%$ \\
\hline Forma clínica $\left(n^{0}=574\right)$ & & & Índice baciloscópico $\left(\mathrm{n}^{0}=436\right)$ & & \\
\hline dimorfa & 478 & 81,8 & negativo $(=0)$ & 321 & 73,6 \\
\hline virchoviana & 99 & 17,0 & positivo $(>0)$ & 115 & 26,4 \\
\hline outras formas & 7 & 1,2 & Nervo ulnar lesado $\left(n^{0}=473\right)$ & & \\
\hline Tempo de tratamento $\left(\mathrm{n}^{0}=525\right)^{*}$ & & & não & 309 & 65,3 \\
\hline$<24$ meses & 184 & 35,0 & $\operatorname{sim}$ & 164 & 34,7 \\
\hline$\geq 24$ meses & 341 & 65,0 & Nervo tibial lesado $\left(\mathrm{n}^{0}=473\right)$ & & \\
\hline Surto reacional $\left(\mathrm{n}^{\underline{0}}=521\right)$ & & & não & 265 & 56,0 \\
\hline não & 422 & 81,0 & $\operatorname{sim}$ & 208 & 44,0 \\
\hline $\operatorname{sim}$ & 99 & 19,0 & Grau de incapacidade na $1^{\text {a }}$ avaliação $\left(n^{0}=584\right)$ & & \\
\hline Tipo de surto $\left(n^{0}=99\right)$ & & & 0 & 369 & 63,2 \\
\hline surto tipo I & 34 & 34,3 & 1 & 110 & 18,8 \\
\hline surto tipo II & 47 & 47,5 & 2 & 105 & 18,0 \\
\hline surto tipos I e II & 18 & 18,2 & Grau de incapacidade na alta $\left(\mathrm{n}^{0}=466\right)$ & & \\
\hline Neurite $\left(\mathrm{n}^{\mathrm{0}}=529\right)$ & & & 0 & 350 & 75,1 \\
\hline não & 248 & 46,9 & 1 & 68 & 14,6 \\
\hline $\operatorname{sim}$ & 281 & 53,1 & 2 & 48 & 10,3 \\
\hline Maior dose de prednisona utilizada $\left(\mathrm{n}^{0}=529\right)$ & & & Tratamento fisioterápico $\left(\mathrm{n}^{0}=520\right)$ & & \\
\hline não usou & 248 & 46,9 & orientações & 345 & 66,3 \\
\hline 20 a $30 \mathrm{mg}$ & 100 & 18,9 & exercícios e orientações & 76 & 14,6 \\
\hline 40 a $50 \mathrm{mg}$ & 120 & 22,7 & imobilizações & 34 & 6,5 \\
\hline$\geq 60 \mathrm{mg}$ & 61 & 11,5 & outros & 65 & 12,5 \\
\hline
\end{tabular}

Fonte: Centro de Saúde Barreiro, Belo Horizonte/Minas Gerais, 2006.

* a variável tempo de tratamento foi categorizada pela mediana. 
26\% apresentavam o índice baciloscópico (IB) positivo. Toda a amostra recebeu acompanhamento fisioterapêutico, sendo que $14,6 \%$ da amostra receberam tratamento fisioterápico com exercícios e orientações, $66,3 \%$ orientações/educação em saúde, 6,5\% necessitaram de algum tipo de imobilização e 12,5\% necessitaram de órteses funcionais/palmilhas e/ou férulas. $\mathrm{E}$ comparando o percentual do grau de incapacidade da primeira avaliação (admissão) com a alta, observa-se aumento do grau de incapacidade zero no momento da alta $(75,1 \%)$.

Os resultados da análise de sobrevida (tábua de vida) estão apresentados na Tabela 2 . Percebe-se que no final do período de observação (24 meses), ainda havia 242 (46\%) indivíduos livres de neurite; no intervalo de tempo de oito a 11,9 meses, a probabilidade de um paciente ainda se encontrar sem neurite foi de 52\%. 0 gráfico de sobrevida pode ser visto na Figura 1.

A análise univariada por meio do modelo de Cox, para demonstrar os fatores associados ao aparecimento da neurite, está apresentada na Tabela 3. As variáveis que se mostraram associadas com a ocorrência da neurite, consideradas no modelo multivariado foram: grau de incapacidade na admissão, idade, forma clínica, índice baciloscópico e número de nervos acometidos. As variáveis ocupação, sexo e região de procedência não foram significativas.

A Tabela 4 mostra o modelo multivariado de Cox, com os respectivos HR (Hazard ratio) e intervalos de confiança. As variáveis que permaneceram no modelo final de Cox foram 0 grau de incapacidade na entrada e índice baciloscópico. Assim, quem teve grau de incapacidade 1 (um) na admissão tem um risco 2,2 vezes maior de ter neurite do que aqueles que foram classificados como grau de incapacidade 0. Já quem tem grau de incapacidade 2 (dois) tem um risco 1,6 vezes maior comparado com quem teve grau de incapacidade 0 (zero). Os pacientes que têm índice baciloscópico positivo têm um risco quase duas vezes maior de ter neurite do que aqueles que tiveram índice baciloscópico negativo. Os respectivos gráficos podem ser vistos nas Figuras 2 A e B.

Tabela 2 - Tábua de vida para tempo até a ocorrência de neurite ( $\left.n^{0}=523\right)$.

\begin{tabular}{lcccccccc}
\hline $\begin{array}{l}\text { Tempo } \\
(\text { meses })\end{array}$ & $\begin{array}{c}\text { Indivíduos } \\
\left(\mathrm{n}^{\circ}\right)\end{array}$ & $\begin{array}{c}\text { Censuras } \\
\left(\mathrm{n}^{\mathrm{o}}\right)\end{array}$ & $\begin{array}{c}\text { Expostos } \\
\text { ao risco } \\
\left(\mathrm{n}^{\mathrm{o}}\right)\end{array}$ & $\begin{array}{c}\text { Ocorrências } \\
\text { do evento } \\
\left(\mathrm{n}^{\mathrm{o}}\right)\end{array}$ & $\begin{array}{c}\text { Ocorrência } \\
\text { do evento } \\
\text { (proporção) }\end{array}$ & $\begin{array}{c}\text { Sobrevida } \\
\text { (proporção) }\end{array}$ & $\begin{array}{c}\text { Sobrevida } \\
\text { acumulada }\end{array}$ & $\begin{array}{c}\text { Standard } \\
\text { error }\end{array}$ \\
\hline $0-3,9$ & 523 & 0 & 523 & 164 & 0,31 & 0,69 & 0,69 & 0,02 \\
$4-7,9$ & 359 & 0 & 359 & 56 & 0,16 & 0,84 & 0,58 & 0,02 \\
$8-11,9$ & 303 & 0 & 303 & 30 & 0,10 & 0,90 & $\mathbf{0 , 5 2}$ & 0,02 \\
$12-15,9$ & 273 & 0 & 273 & 13 & 0,05 & 0,95 & 0,50 & 0,02 \\
$16-19,9$ & 260 & 0 & 260 & 8 & 0,03 & 0,97 & 0,48 & 0,02 \\
$20-23,9$ & 252 & 0 & 252 & 8 & 0,03 & 0,97 & 0,47 & 0,02 \\
$\geq 24$ & 244 & 242 & 123 & 2 & 0,02 & 0,98 & $\mathbf{0 , 4 6}$ & 0,02 \\
\hline
\end{tabular}

Fonte: Centro de Saúde Barreiro, Belo Horizonte/Minas Gerais, 2006

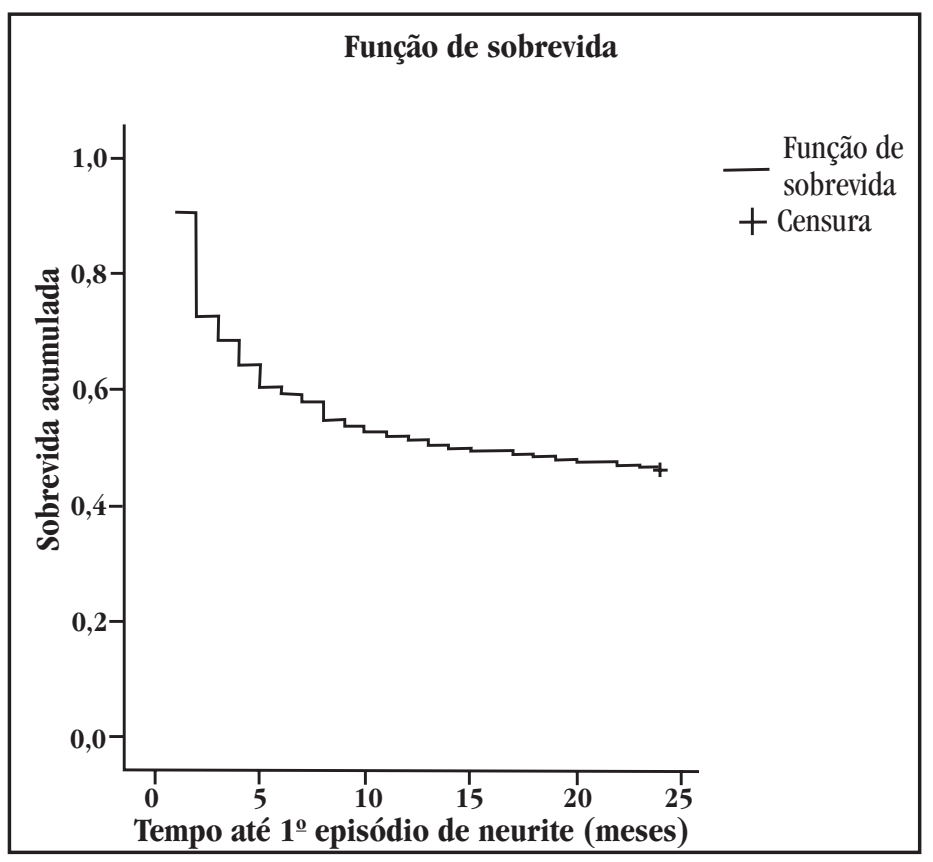

Fonte: Centro de Saúde Barreiro, Belo Horizonte/Minas Gerais, 2006.

Figura 1 - Função de sobrevida obtida por meio do Método de Kaplan-Meier. 
Tabela 3 - Análise da ocorrência de neurite por variável sociodemográfica e clínica.

\begin{tabular}{|c|c|c|c|c|c|c|}
\hline \multirow[t]{2}{*}{ Variáveis sociodemográficas e clínicas } & \multicolumn{3}{|c|}{ Eventos } & \multirow[t]{2}{*}{ HR } & \multirow{2}{*}{$\begin{array}{c}\text { IC }(95 \%) \\
\text { para HR }\end{array}$} & \multirow[t]{2}{*}{ Valor $\mathrm{p}$} \\
\hline & $\mathrm{n}^{\mathrm{o}}$ total & (neurite) & $\%$ & & & \\
\hline \multicolumn{7}{|l|}{ Ocupação } \\
\hline serviços, vendedores do comércio & 89 & 49 & 55,1 & 0,91 & $(0,62-1,36)$ & 0,280 \\
\hline produção de bens e serviços industriais & 81 & 44 & 54,3 & 0,95 & $(0,64-1,43)$ & \\
\hline aposentado & 34 & 21 & 61,8 & 1,04 & $(0,62-1,73)$ & \\
\hline do lar & 71 & 37 & 52,1 & 0,87 & $(0,57-1,33)$ & \\
\hline estudante & 46 & 17 & 37,0 & 0,52 & $(0,30-0,90)$ & \\
\hline outros & 87 & 50 & 57,5 & 1,00 & - & \\
\hline \multicolumn{7}{|l|}{ Grau de incapacidade na entrada } \\
\hline 0 & 327 & 145 & 44,3 & 1,00 & & $<0,001$ \\
\hline 1 & 99 & 77 & 77,8 & 2,36 & $(1,79-3,12)$ & \\
\hline 2 & 88 & 57 & 64,8 & 1,75 & $(1,29-2,38)$ & \\
\hline \multicolumn{7}{|l|}{ Sexo } \\
\hline feminino & 267 & 141 & 52,8 & 1,00 & & 0,708 \\
\hline masculino & 256 & 140 & 54,7 & 1,05 & $(0,83-1,32)$ & \\
\hline \multicolumn{7}{|l|}{ Idade (anos) } \\
\hline$<42$ & 217 & 109 & 50,2 & 1,00 & & 0,047 \\
\hline$\geq 43$ & 207 & 126 & 60,9 & 1,30 & $(1,01-1,68)$ & \\
\hline \multicolumn{7}{|l|}{ Forma clínica } \\
\hline dimorfa & 432 & 217 & 50,2 & 1,00 & & 0,002 \\
\hline outras formas & 83 & 57 & 68,7 & 1,58 & $(1,18-2,11)$ & \\
\hline \multicolumn{7}{|l|}{ Índice baciloscópico } \\
\hline negativo $(=0)$ & 315 & 145 & 46,0 & 1,00 & & $<0,001$ \\
\hline positivo $(>0)$ & 112 & 86 & 76,8 & 2,17 & $(1,66-2,84)$ & \\
\hline \multicolumn{7}{|l|}{ Número de nervos acometidos } \\
\hline$<3$ & 332 & 167 & 50,3 & 1,00 & & $<0,001$ \\
\hline$\geq 3$ & 131 & 96 & 73,3 & 1,83 & $(1,42-2,35)$ & \\
\hline \multicolumn{7}{|l|}{ Região de procedência } \\
\hline Barreiro (BH) & 190 & 107 & 56,3 & 1,15 & $(0,88-1,51)$ & 0,561 \\
\hline Contagem & 127 & 67 & 52,8 & 1,02 & $(0,75-1,39)$ & \\
\hline outros & 199 & 104 & 52,3 & 1,00 & & \\
\hline
\end{tabular}

Fonte: Centro de Saúde Barreiro, Belo Horizonte/Minas Gerais, 2006.

HR: Hazard ratio, IC: intervalo de confiança 95\%.
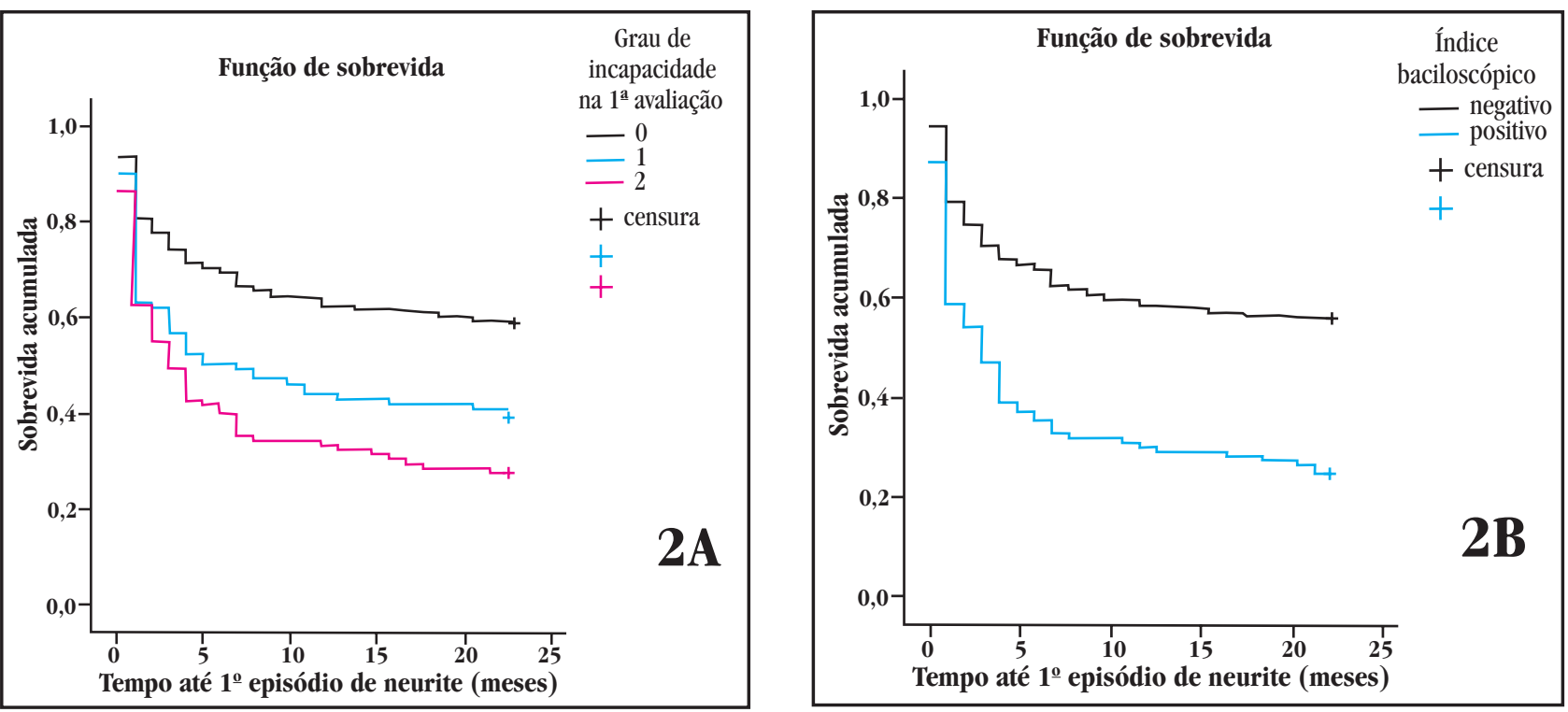

Fonte: Centro de Saúde Barreiro, Belo Horizonte/Minas Gerais, 2006.

Figuras $2 A$ e B - Função de sobrevida (Kaplan-Meier) das variáveis que permaneceram no modelo final de Cox (grau de incapacidade e índice baciloscópico, respectivamente). 
Tabela 4 - Modelo multivariado de Cox para o tempo até ocorrência do 1 opisódio de neurite.

\begin{tabular}{lccc}
\hline Variáveis & HR & $\begin{array}{c}\text { IC (95\%) para } \\
\text { HR }\end{array}$ & Valor p \\
\hline $\begin{array}{l}\text { Grau de incapacidade na entrada } \\
0\end{array}$ & 1,00 & & \\
1 & 2,17 & $(1,59-2,95)$ & $<0,001$ \\
2 & 1,64 & $(1,17-2,32)$ & \\
Índice baciloscópico & & & \\
$\quad$ negativo $(=0)$ & 1,00 & & $<0,001$ \\
$\quad$ positivo $(>0)$ & 1,93 & $(1,46-2,53)$ & \\
\hline
\end{tabular}

Fonte: Centro de Saúde Barreiro, Belo Horizonte/Minas Gerais, 2006 HR: Hazard ratio, IC: intervalo de confiança 95\%.

\section{DISCUSSÃo}

A literatura apresenta resultados percentuais variados quanto à ocorrência dos episódios de surtos reacionais, neurites e incapacidades $^{78912}$ No presente estudo, a neuropatia ocorreu em mais da metade dos pacientes e o quadro reacional em $19 \%$ da amostra, caracterizando risco elevado de deficiências e incapacidades. Na presente pesquisa os principais fatores associados à ocorrência de neurite durante o tratamento foi 0 grau de incapacidade apresentado no início do tratamento e a positividade do IB, confirmando a importância da busca ativa e do diagnóstico precoce. Os pacientes que iniciaram o tratamento com o grau de incapacidade 1 (um) tiveram maior chance de desenvolver a neuropatia em um tempo mais precoce, seguido do grau de incapacidade 2 (dois). Esse fato pode ser explicado pela potencial instabilidade neurológica dos pacientes que são classificados no grau 1 (um) de incapacidade, podendo ocorrer com maior intensidade e frequiência à piora ou a melhora na sua evolução. Já os pacientes classificados no grau de incapacidade 2 (dois) apresentam danos neurais com seqüelas geralmente instaladas e definitivas, o que reduz as chances de ocorrer neuropatias agudas, pois os nervos periféricos já podem apresentar danos irreversíveis. 0 grau de incapacidade zero (0) e o IB negativo refletem a precocidade do diagnóstico e menor chance de danos neurais primários devido à possibilidade de monitoramento neurológico, diagnósticos rápidos e tratamentos adequados para os acometimentos neurais. Sharma e cols ${ }^{16}$ afirmam que, quando o tratamento foi iniciado em estágios precoces, as chances de recuperação pareceram melhores.

Os pacientes com o IB positivo também apresentaram maior chance de apresentarem neurite mais precocemente. Selvaraj e cols ${ }^{15}$, em estudo realizado na Índia, com 1.262 pacientes, também encontraram associação entre 0 IB positivo e a presença de deformidades. Esses resultados confirmam a necessidade do diagnóstico precoce da hanseníase e tratamento imediato para se evitar os danos neurais. Ou seja, quanto menor o grau de incapacidade no início do tratamento e a não-positividade do IB, maior a possibilidade de se evitar deformidades e incapacidades ${ }^{14}{ }^{15}$. Sharma e cols ${ }^{16}$ não encontraram associação entre o grau de incapacidade e carga bacilar. Porém, o estudo afirma que o único fator que parece suportar alguma relação com a recuperação dos danos primários foi o estágio precoce de incapacidades durante o início da PQT. No presente estudo, encontrou-se associação entre a carga bacilar e à ocorrência de neurite, demonstrando que a positividade bacilar pode desencadear precocemente os quadros de acometimento neural, podendo levar às deficiências e incapacidades, se não tratado adequadamente. 0 estudo de Meima e cols ${ }^{12}$ afirma que os dois fatores de risco mais associados à deformidade foram a demora entre o tempo de aparecimento dos sintomas e o diagnóstico, bem como a idade.

É interessante perceber também que o tempo máximo até a ocorrência da neurite neste estudo foi de 24 meses, com $48 \%$ dos casos ocorrendo até o $12^{\circ}$ mês de tratamento. Sendo, portanto, indicado neste período mais crítico, o acompanhamento neurológico dos pacientes, de maneira regular e frequiente. Contudo, o estudo demonstrou também que há risco de desenvolver o quadro de neurite, com possibilidades de novas incapacidades, principalmente nos 12 meses consecutivos. Considerando a alta dos multibacilares, atualmente, com 12 doses supervisionadas de PQT, os resultados indicam que seria aconselhável o monitoramento neurológico também após a alta ${ }^{45}$.

Em uma coorte prospectiva realizada em Bangladesh', 2.510 pacientes foram acompanhados no período de dois anos. Destes, 166 desenvolveram novas incapacidades. Os autores utilizaram o modelo mutivariado de Cox para predizer incapacidade, demonstrando que os principais fatores de risco para novas incapacidades foram classificação em multibacilar (MB) e paucibacilar (PB) e a presença de danos ao nervo na admissão ao tratamento. Os autores estratificaram os resultados em grupos de risco para novas deformidades, ou seja, risco leve, moderado e alto. Pacientes MB com disfunção do nervo periférico na admissão apresentaram $65 \%$ de risco (alto) de desenvolver novos danos no nervo até 12 meses após a alta. Esse trabalho demonstrou a necessidade de acompanhamento após a alta, dependendo do grupo de risco do paciente, considerando essa possibilidade de ocorrência de novos danos neurais principalmente nos primeiros 12 meses após a alta. Esses resultados estão de acordo com o presente estudo, confirmando a importância destas evidências.

Sendo a neurite um quadro frequiente, o monitoramento neural por meio da avaliação neurológica, deve ser prática inquestionável, principalmente para aqueles pacientes que associam baciloscopia positiva e grau de incapacidade 1 (um) no momento do diagnóstico de hanseníase, promovendo assim intervenções precoces e adequadas. 0 aumento do percentual de grau zero no momento da alta, encontrado na presente amostra, demonstra que houve critérios adequados de monitoramento e intervenções pela equipe que assistia os pacientes.

Concluiu-se que o grau de incapacidade no momento do diagnóstico e a carga bacilar se associaram fortemente à ocorrência de neurite, confirmando a necessidade do diagnóstico precoce da hanseníase, bem como do acompanhamento neurológico regular desses pacientes para a detecção precoce das neurites, possibilitando intervenções medicamentosas e não medicamentosas por meio de técnicas de prevenção de incapacidades, para se evitar as deformidades. 
0 estudo demonstrou também que os primeiros 12 meses após o diagnóstico são os principais no controle dos quadros de neuropatia, podendo ocorrer o evento até 24 meses, sendo, portanto necessário um prolongamento do acompanhamento neurológico, mesmo após a alta medicamentosa.

\section{REFERÊNCIAS}

1. Birdi TJ, Antia NH. Mechanisms involved in peripheral nerve damage in leprosy with special reference to insights obtained from in vitro studies and the experimental mouse model. International Journal of Leprosy 71: 345-354, 2003.

2. Carvalho MS, Andreozzi VL, Codeço CT, Barbosa MTS, Shirnakura SE. Análise de sobrevida. Teoria e Aplicações em Saúde. Editora Fundação Oswaldo Cruz, Rio de Janeiro, 2005.

3. Charosky CB, Gatti JC, Cardama JE. Neuropathies in Hansen's disease (editorials). International Journal of Leprosy 51: 576-586, 1983.

4. Croft RP, Nicholls PG, Steyerberg EW, Richardua JH, Smith WCS. A clinical prediction rule for nerve-function impairment in leprosy patients. The Lancet 355:1603-1606, 2000

5. Croft RP, Richardus JH, Nicholls PG, Smith WCS. Nerve function impairment in leprosy: design, methodology, and intake status of a prospective cohort study of 2664 new leprosy cases in Bangladesh (The Bangladesh Acute Nerve Damage Study). Leprosy Review 70: 140-159, 1999.

6. Deepak S. Answering the rehabilitation needs of leprosy affected persons in integrated setting through primary health care services and community based rehabilitation. Indian Journal Leprosy 75:127-142, 2003.

7. Foss NT, Souza CS, Gourlart IMB, Gonçalves HS, Virmond M. Hanseníase: episódios reacionais. Projeto diretrizes. Associação Médica Brasileira e Conselho Federal de Medicina. 2003.

8. Guocheng Z, Wenzhong L, Liangbin Y, Zhongmin Y, Yiangsheng C, Tisheng Z, Ganyun Y. An epidemiological survey of deformities and disabilities among 14.257 cases of leprosy in 11 counties. Leprosy Review 64: 143-149, 1993.
9. Kumar B, Dogra S, Kaur I. Epidemiological characteristics of leprosy reactions: 14 years experience from North India. International Journal of Leprosy 72:125-133, 2004.

10. Kumar V, Sengupta U. Ultrastructural Study of Schwann Cells and Endotelial Cells in the Patogenesis of Leprous Neuropathy. International Journal of Leprosy 71:328-340, 2003.

11. Makino M, Maeda Y, Ishii N. Immunostimulatory activity of major membrane protein-II from Mycobacterium leprae. Cellular Immunology 233:53-60, 2005.

12. Meima A, Sauderson PR, Gebre S, Desta K, Van Oortmarssen GJ, Hablema JDF. Factors associated with impairments in new leprosy patients: the AMFES cohort. Leprosy Review 70:189-203, 1999.

13. Rambukkana A. Molecular basis for the peripheral nerve predilection of Mycobacterium leprae. Current Opinion in Microbiology 4:21-27, 2001.

14. Saunderson P, Gebre S, Desta K, Byass P, Lockwood DNJ. The pattern of leprosy-related neuropathy in the AMFES patients in Ethiopia: definitions, incidence, risk factors and autcome. Leprosy Review 71: 285-308, 2000.

15. Selvaraj G, Prabakar N, Muliyil J, Martin G. Incidence of disabilities among multibacillary cases after initiation of multidrug therapy and factors associated with the risk of developing disabilities. Indian Journal Leprosy 70 (suppl):11-16, 1998.

16. Sharma P, Beena HK, Kaur H, Narayan R. Disabilities in multibacilary leprosy patients: before, during and after multidrug therapy. Indian Journal Leprosy 68:127-136, 1996.

17. Shetty VP, Mistry NF, Antia NH. Current understanding of leprosy as a peripheral nerve disorder: significance of involvement of peripheral nerve in leprosy. Indian Journal Leprosy 72:339-350, 2000.

18. Spierings E, Boer TD, Zulianello L, Ottenhoff THM. Novel mechanisms in the immunopathogenesis of leprosy nerve damage: the role of Schwann cells, T cells and Mycobacterium Leprae. Immunology and Cell Biology 78:349-355, 2000.

19. Statistical Package for the Social Sciences incorporation. SPSS for Windows. Release 12.0. SPSS Inc., Chicago, 2003.

20. Van Brakel WH. Detecting peripheral nerve damage in the field our tools in 2000 and beyond. Indian Journal Leprosy 72:47-64, 2000.

21. Van Brakel WH. Peripheral neuropathy in leprosy and its consequences. Leprosy Review 71: S146-S153, 2000. 